ANL-HEP-PR-00-093

\title{
Compatibility of various approaches to heavy-quark fragmentation
}

\author{
G. T. Bodwin and B. W. Harris \\ High Energy Physics Division, Argonne National Laboratory, Argonne, Illinois 60439
}

(December 2000)

\begin{abstract}
We find that the definition of the heavy-quark fragmentation function given by Jaffe and Randall differs by a factor of the longitudinal-momentum fraction $z$ from the standard Collins-Soper definition. Once this factor is taken into account, the explicit calculation of Braaten et al. is found to be in agreement with the general analysis of Jaffe and Randall. We also examine the model of Peterson et al. for heavy-quark fragmentation and find that the quoted values of the width and of the value of $z$ at the maximum are in error. The corrected values are in agreement with the analysis of Jaffe and Randall.

PACS numbers: 12.39.Hg, 13.87.Fh, 14.40.Lb, 14.40.Nd
\end{abstract}

\section{INTRODUCTION}

There does not yet exist a complete first-principles calculation of the nonperturbative transition of a parton (emerging from a high energy scattering process) to a hadron. Nevertheless, a rigorous formal description of fragmentation functions in terms of operator matrix elements has been available for some time [1]. Additionally, fragmentation functions are an integral part of present day phenomenology.

When the parton involved in the fragmentation is a relatively heavy charm or beauty quark, there are simplifications in the treatment of fragmentation functions that allow one to make additional theoretical progress. It is the case of heavy-quark fragmentation that we consider here.

A theoretical description of fragmentation that is based on the operator-matrix-element definition of the fragmentation function in heavy-quark effective theory (HQET) [2] has been presented by Jaffe and Randall [3]. They show that the heavy-quark expansion of the fragmentation function must take a specific form that depends only on certain combinations of variables. In this paper, we point out that the Jaffe-Randall definition of the fragmentation function differs from the standard Collins-Soper definition by a factor of the longitudinalmomentum fraction $z$ and that this difference is crucial to the proper interpretation of the Randall-Jaffe form.

The work of Braaten et al. 浊 models the heavy-quark fragmentation function with a fixed-order perturbative calculation in the context of HQET. The results of this calculation appear, at first sight, to be incompatible with the Jaffe-Randall form. However, after accounting for the difference between the Jaffe-Randall definition of the fragmentation function and the Collins-Soper definition, which is used by Braaten et al., we find agreement be- tween the explicit calculation and the Jaffe-Randall form.

Perhaps the most widely-used parameterization for fragmentation functions is that of the model of Peterson et al. [5], which is based on old-fashioned perturbation theory. The Peterson parameterization has been fit to charm and beauty production data [6] and subsequently used as input in making predictions for hadron-, photo-, and electro-production of charm and beauty [7].

It has been suggested that the Peterson et al. parameterization is incompatible the Jaffe-Randall form. In particular, it has been reported that the width of the fragmentation function is different in the two approaches [3]. We find that the width and value of $z$ corresponding to the maximum are reported incorrectly in the original Peterson et al. paper [5]. The corrected values are compatible with the Jaffe-Randall form.

In Sec. II, we give an overview of the general HQET analysis of Jaffe and Randall. We discuss the compatibility of the explicit calculation of Braaten et al. with the Jaffe-Randall form in Sec. III. Sec. IV contains a description of the model of Peterson et al. and a discussion of its relation to the Randall-Jaffe form. Our findings are summarized in Sec. V.

\section{THE HQET ANALYSIS OF JAFFE AND RANDALL}

We now discuss the work of Jaffe and Randall [3], which provides a QCD-based interpretation of heavyquark fragmentation in terms of the heavy-quark mass expansion. We begin with the standard Collins-Soper [1] definition of the fragmentation function for a heavy quark into a heavy hadron: 


$$
\begin{aligned}
\hat{f}\left(z, \mu^{2}\right) & =\frac{z^{d-3}}{4 N_{c}}\left[\int \frac{d \lambda}{2 \pi} e^{i \lambda / z} \operatorname{Tr} \not h\right. \\
& \left.\times\left\langle 0|h(\lambda n)| H^{\prime}(P)\right\rangle\left\langle H^{\prime}(P)|\bar{h}(0)| 0\right\rangle\right]
\end{aligned}
$$

where the trace is over color and Dirac indices, $N_{c}$ is the number of colors, $h(x)$ is the heavy-quark field at space-time position $x, P$ is the four-momentum of the heavy hadron, $n$ is defined by $n^{2}=0$ and $n \cdot P=1$, and $z=n \cdot P / n \cdot k$, where $k$ is the heavy-quark momentum. The state $\left|H^{\prime}(P)\right\rangle$ consists of the heavy hadron plus any number of additional hadrons. The matrix element is understood to be evaluated in the light-cone gauge $n$. $A=0$. Eqn. (11) is valid in $d$ dimensions. However, in discussing the Jaffe-Randall analysis, we specialize to the case of four dimensions.

The definition (11) is normalized such that $\hat{f}\left(z, \mu^{2}\right)$ times the parton-level cross section reproduces the full QCD cross section, differential in $z$, in the collinear limit. By "collinear limit" we mean the limit in which $\sqrt{k^{2}}$ and the transverse component (relative to $P$ ) of the 3 momentum of $k$ are neglected in comparison to the longitudinal component of the 3 -momentum of $k$. This normalization ensures that $\hat{f}\left(z, \mu^{2}\right)$ is a probability distribution in $z$ (Ref. [1]).

\footnotetext{
* The normalization on the right side of Eq. (1) can be understood as follows. For simplicity, we consider a frame in which $P=\left(P^{+}, P^{2} / P^{+}, 0_{\perp}\right)$ has no transverse component. $k=\left(k^{+}, k^{-}, k_{\perp}\right)$, with $P^{+}=z k^{+}$. In this frame, $n=\left(0,1 / P^{+}, 0_{\perp}\right)$ and $\lambda=P^{+} x^{-}$, where $x$ is the spacetime separation of the quark and antiquark fields. In the collinear limit, $k^{-}$and $k_{\perp}$ are neglected in comparison with $k^{+}$. In that limit, the full QCD cross section is reproduced by the parton-level cross section, times the Fourier transform in brackets in Eq. (1), times a normalization factor. This normalization factor includes $1 /\left(2 P^{+}\right)$for the normalization of the hadron state, $2 k^{+}$for the normalization of the quark state in the parton-level cross section, $1 / 4$ from the Fierz re-arrangement $I_{\alpha \alpha^{\prime}} I_{\beta \beta^{\prime}} \rightarrow(1 / 4) \gamma_{\alpha \beta}^{+} \gamma_{\alpha^{\prime} \beta^{\prime}}^{-}$that factors the Dirac indices, $1 / N_{c}$ from the Fierz re-arrangement $I_{a a^{\prime}} I_{b b^{\prime}} \rightarrow\left(1 / N_{c}\right) I_{a b} I_{a^{\prime} b^{\prime}}$ that factors the color indices, and $1 / k^{+}$for the quark sum-over-spinors projector $\left(\gamma \cdot k \approx \gamma^{-} k^{+}\right)$ in the parton-level cross section. The full QCD cross section is differential in the longitudinal momentum of the final-state hadron. Since $d P^{+}=-k^{+} d z$, we obtain an additional factor of $k+$ on conversion to a cross section that is differential in $z$. In the full QCD cross section, there is also an integral over the transverse momentum of the hadron. This integration corresponds, in the frame in which the hadron has zero transverse momentum, to an integration with respect to $d^{d-2}\left(z k_{\perp}\right)$ (Ref. 迎). The conversion to an integration with respect to $d^{d-2} k_{\perp}$ yields a factor $z^{d-2}$. (The integration over all $k_{\perp}$ corresponds to taking the quark and antiquark fields at transverse separation $x_{\perp}=0$.) Altogether, we obtain a factor $z^{d-3} /\left(4 N_{c}\right)$, which is just the coefficient of the Fourier transform in Eq. (1).
}

We note that the definition (11) contains a factor $z$ (in four dimensions) relative to the definition of the fragmentation function used by Jaffe and Randall [3]. This factor $z$ will be important in comparing with the work of Braaten et al. below.

The analysis of Eq. (11) in HQET proceeds as follows. First, following the standard method for obtaining the heavy-quark-mass expansion, one decomposes the field $h(x)$ into the sum of a large component $h_{v}(x)$ and a small component $\underline{h}_{v}(x)$ :

$$
\begin{aligned}
& h_{v}(x)=e^{-i m_{Q} v \cdot x} P_{+} h(x) \\
& \underline{h}_{v}(x)=e^{-i m_{Q} v \cdot x} P_{-} h(x),
\end{aligned}
$$

with $m_{Q}$ the heavy-quark mass, $P_{ \pm}=(1 \pm \psi) / 2$, and $v$ the hadron's four-velocity. The leading term in the mass expansion of $f\left(x, \mu^{2}\right)$ is contained in the large-large combination of fields:

$$
\begin{aligned}
\hat{f}\left(z, \mu^{2}\right) & =\frac{z}{4 N_{c}} \int \frac{d \lambda}{2 \pi} e^{i \lambda / z} \operatorname{Tr} \not h e^{-i m_{Q} \lambda n \cdot v} \\
& \times\left\langle 0\left|P_{+} h(\lambda n)\right| H^{\prime}(P)\right\rangle\left\langle H^{\prime}(P)\left|\overline{P_{+} h(0)}\right| 0\right\rangle \\
& +\ldots .
\end{aligned}
$$

Here, and throughout this paper, we use the ellipsis to denote terms of higher order in the hadron-mass expansion.

In Ref. [3], it is argued that the matrix element in Eq. (4) is a dimensionless function $\mathcal{F}(\lambda \delta)$. This function may be written in terms of its Fourier transform:

$$
\mathcal{F}(\lambda \delta)=2 \int_{-\infty}^{\infty} d \alpha e^{-i \alpha \lambda \delta} a(\alpha)
$$

where

$$
\delta=1-m_{Q} / m_{H},
$$

and $m_{H}$ is the hadron mass. Inserting Eq. (5) into Eq. (4), one can evaluate the integral, with the result

$$
\hat{f}\left(z, \mu^{2}\right)=\frac{z}{\delta} \hat{a}\left(\frac{1 / z-m_{Q} / m_{H}}{\delta}\right)+\ldots .
$$

A more complete analysis in Ref. [3] also yields the nextto-leading term in the hadron-mass expansion:

$$
\hat{f}\left(z, \mu^{2}\right)=z\left[\frac{1}{\delta} \hat{a}(y)+\hat{b}(y)+\cdots\right],
$$

where $y=\left(1 / z-m_{Q} / m_{H}\right) / \delta$. The analysis in Ref. [3] does not yield a precise prediction for the functional form of $a$ and $b$, but some general properties may be deduced. The function $a$ describes, in the limit of infinite heavyquark mass, the effects of binding in the heavy hadron on the heavy-quark momentum distribution. For a free heavy quark, $a(y)$ would be a $\delta$-function at $y=1$. In a heavy hadron, the binding smears the heavy-quark momentum distribution. It can be shown [3] that the distribution has a width 


$$
\Delta z \sim \delta
$$

and a maximum at $z=z_{\max }$, where

$$
1-z_{\max } \sim \delta
$$

\section{A PERTURBATIVE MODEL}

Braaten et al. [1] present a QCD-inspired model for the fragmentation of a heavy quark into an $S$-wave lightheavy meson. In this model, the fragmentation function is computed in perturbative QCD (at Born level) in an expansion in inverse powers of the heavy-quark mass.

Braaten et al. define the fragmentation function as the collinear limit of the ratio of the cross section for producing a hadron to the cross section for producing a quark. As we explained in Sec. II, this definition is equivalent to the Collins-Soper definition (11) and leads to a fragmentation function that is a probability distribution in $z$.

For the projection of the $Q q$ state onto the meson, Braaten et al. take the standard nonrelativistic-boundstate expression. For example, in the case of a ${ }^{1} S_{0}$ meson, they assume the Feynman rule for the $Q q H$ vertex to be

$$
\frac{\delta_{i j}}{\sqrt{3}} \frac{R(0) \sqrt{m_{H}}}{\sqrt{4 \pi}} \gamma_{5}(1+\psi) / 2,
$$

where $R(0)$ is the radial wave function at the origin. From the terms of leading order in the heavy-quark mass expansion, they obtain, in the case of a ${ }^{1} S_{0}$ meson,

$$
\begin{aligned}
\hat{f} & \approx N\left[\frac{1}{\delta} \frac{(1-y)^{2}}{y^{6}}\left(3 y^{2}+4 y+8\right)\right. \\
& \left.-\frac{(1-y)^{3}}{y^{6}}\left(3 y^{2}+4 y+8\right)\right],
\end{aligned}
$$

where $N=2 \alpha_{s}^{2}|R(0)|^{2} /\left(81 \pi m_{q}^{3}\right)$.

At first glance, this result may seem to contradict the Jaffe-Randall analysis, which shows that the terms of leading order in the heavy-quark mass expansion give a contribution that is contained entirely in the function $a(y)$ in Eq. (8). However, the factor $z$ in the definition of the fragmentation function (i1) is crucial here. From the definitions of $y$ and $\delta$, we have $z=1 /[1-\delta(1-y)]$, and, so, we can re-write Eq. (11) as

$$
\hat{f} / z \approx \frac{N}{\delta} \frac{(1-y)^{2}}{y^{6}}\left(3 y^{2}+4 y+8\right),
$$

which is of the form of $a(y)$ in Eq. (8).

\section{PETERSON FRAGMENTATION}

Finally, we examine the model of Peterson et al. [5] for the fragmentation of a fast-moving heavy quark $Q$ with mass $m_{Q}$ into a heavy hadron $H$ (consisting of $Q \bar{q}$ ) with mass $m_{H}$ and a light quark $q$ with mass $m_{q}$. The basic assumption in this model is that the amplitude for the fragmentation is proportional to $1 /(\Delta E)$, where $\Delta E=E_{H}+E_{q}-E_{Q}$ is the energy denominator for the process in old-fashioned perturbation theory. It follows that the probability for the transition $Q \rightarrow H+q$ is proportional to $1 /(\Delta E)^{2}$. Taking the heavy quark's momentum to define the longitudinal axis, one can express $\Delta E$ in terms of the magnitude of the heavy quark's momentum $P_{Q}$, the fraction $z$ of the heavy quark's momentum that is carried by the heavy hadron, and the transverse momentum $p_{\perp}$ of the heavy hadron or the light quark:

$$
\begin{aligned}
\Delta E & =\sqrt{m_{H}^{2}+p_{\perp}^{2}+z^{2} P_{Q}^{2}}+\sqrt{m_{q}^{2}+p_{\perp}^{2}+(1-z)^{2} P_{Q}^{2}} \\
& -\sqrt{m_{Q}^{2}+P_{Q}^{2}} \\
& \approx \frac{m_{H}^{2}+p_{\perp}^{2}}{2 z P_{Q}}+\frac{m_{q}^{2}+p_{\perp}^{2}}{2(1-z) P_{Q}}-\frac{m_{Q}^{2}}{2 P_{Q}}+\cdots \\
& \approx-\frac{m_{Q}^{2}}{2 P_{Q}}[1-1 / z-\epsilon /(1-z)] .
\end{aligned}
$$

In the last line, we have set $m_{H} \approx m_{Q}$ and neglected $p_{\perp}^{2}$ relative to $m_{Q}^{2}$ and used the definition

$$
\epsilon \equiv\left(m_{q}^{2}+p_{\perp}^{2}\right) / m_{Q}^{2} .
$$

Multiplying $1 /(\Delta E)^{2}$ by a factor $1 / z$ for the longitudinal phase space, one arrives at the following ansatz for the fragmentation function [5]

$$
D_{Q}^{H}(z)=\frac{N}{z[1-1 / z-\epsilon /(1-z)]^{2}},
$$

where the normalization $N$ is fixed by the condition

$$
\sum_{H} \int d z D_{Q}^{H}(z)=1
$$

and the sum extends over all hadrons that contain $Q$.

Contrary to the claims in Ref. [5], we find that $D_{Q}^{H}(z)$ has a maximum at $z=z_{\max }$, with $z_{\max } \approx 1-\sqrt{\epsilon}$, and a width of order $\sqrt{\epsilon}$. Specifically, we find, that the distance between the inflection points of $D_{Q}^{H}(z)$ is $[(8-2 \sqrt{3}) / 3]^{1 / 2} \sqrt{\epsilon}$, and the full width at half maximum is $2 \sqrt{\epsilon}$. In these values of $z_{\max }$ and the width, we have neglected terms of higher order in $\epsilon$.

We can compare the Peterson et al. form with the Randall-Jaffe results by making use of Eqs. (6) and (14), to obtain

$$
\sqrt{\epsilon} \approx \delta .
$$


It has been believed that the shape of the Peterson et al. fragmentation function is incompatible with results obtained from heavy quark effective theory (HQET). However, it follows immediately from Eq. (17) that our results for the width and $z_{\max }$ of the Peterson form are compatible with the Jaffe-Randall constraints (9).

\section{SUMMARY}

We have examined the Randall-Jaffe analysis of the heavy-quark fragmentation function in HQET and found that the Randall-Jaffe definition of the heavy-quark fragmentation function differs from the standard CollinsSoper definition (11) by a factor $z$. This factor is crucial to the interpretation of the fragmentation function as a probability distribution in $z$. We have found that, once this factor has been taken into account, the explicit, perturbative model calculation of Braaten et al. is in agreement with the Randall-Jaffe analysis.

We have also examined the model of Peterson et al. for the heavy-quark fragmentation function. Our results for the width and the value of $z$ corresponding to the maximum of the Peterson fragmentation function differ from the values stated in the Peterson et al. paper [5]. Our values are consistent with constraints from the general analysis of Randall and Jaffe.

\section{ACKNOWLEDGMENTS}

We thank R. Jaffe for confirming the relationship of the Randall-Jaffe definition of the fragmentation function to the Collins-Soper definition. This work was supported by the United States Department of Energy, High Energy Physics Division, under contract W-31-109-Eng-38.

[1] J. C. Collins and D. E. Soper, Nucl. Phys. B194, 445 (1982)

[2] For reviews see B. Grinstein, Ann. Rev. Nucl. Part. Sci. 42, 101 (1992); M. Neubert, Phys. Rept. 245, 259 (1994); A. F. Falk in Proceedings of the XXIVth SLAC Summer Institute on Particle Physics, edited by J. Chan, L. DePorcel and L. Dixon ( NTIS, Springfield, VA, 1997), p. 43.

[3] R. L. Jaffe and L. Randall, Nucl. Phys. B412, 79 (1994).

[4] E. Braaten, K. Cheung, S. Fleming, and T. C. Yuan, Phys. Rev. D 51, 4819 (1995).

[5] C. Peterson, D. Schlatter, I. Schmitt, and P. M. Zerwas, Phys. Rev. D 27, 105 (1983).

[6] P. Nason and C. Oleari, Nucl. Phys. B565, 245 (2000); M. Cacciari and M. Greco, Phys. Rev. D 55, 7134 (1997).

[7] S. Frixione, M. L. Mangano, P. Nason, and G. Ridolfi, Nucl. Phys. B431, 453 (1994); J. Binnewies, B. A. Kniehl,
G. Kramer, Phys. Rev. D 58, 034016 (1998); D 58, 014014 (1998); B. W. Harris and J. Smith, Phys. Rev. D 57, 2806 (1998); S. Frixione, M. Kramer, E. Laenen, Nucl. Phys. B571, 169 (2000). 